\title{
DNA ploidy of cervical epithelial cells should be a cure criterion of high-risk HPV infection in Xinjiang Uygur women
}

This article was published in the following Dove Press journal:

OncoTargets and Therapy

13 April 2015

Number of times this article has been viewed

\author{
Yang-Chun Feng' \\ Jia Yang ${ }^{2}$ \\ Cheng-Ming Liu' \\ Zhen-Zhen Cheng' \\ Yan-Chun Huang' \\ 'Clinical Laboratory Center, \\ ${ }^{2}$ Department of Second Internal \\ Medicine, Tumor Hospital Affiliated \\ to Xinjiang Medical University, \\ Urumqi, People's Republic of China
}

Correspondence: Yan-Chun Huang Clinical Laboratory Center, Tumor Hospital Affiliated to Xinjiang Medical University, No 789

Suzhou Road, Urumqi 8300II, People's Republic of China

Tel +869917819426

Email442531979@qq.com
Background: The Uygur women have the highest incidence of cervical cancer in all Chinese ethnic groups. The research was conducted to explore whether DNA ploidy could be the prognostic indicator of human papillomavirus (HPV) infection in Xinjiang Uygur women.

Methods: Case data and cervical exfoliated cell samples from 326 Uygur women. The DNA ploidy was analyzed by flow cytometry. The flow-through hybridization and gene chip (FHGC) for HPV type test then divided the cases into negative HPV group, non high-risk HPV infection group, single high-risk HPV infection group, and multiple high-risk HPV infection group. Lastly, 113 cases from 273 HPV infection cases were followed up.

Results: The 16-type HPV had the highest rate in all genotypes infection; 16/18-type HPV mixed infection was the most common type in multiple high-risk HPV infection group. Abnormal DNA ploidy happened along with the seriousness of HPV infection. Compared with the HPV negative group, DNA heteroploid appeared 12.750 times and 22.705 times, respectively, in single high-risk HPV and multiple high-risk HPV infection groups. Followed up 1 year later, the DNA index, S-phase cells' peak percentage and heteroploid of cervical exfoliated cells significantly reduced in single and multiple high-risk HPV infection patients, but in nine patients negative HPV infection and DNA heteroploid still existed.

Conclusion: The finally cure criterion of high-risk HPV infection should include the negative HPV test and normal DNA ploidy analysis. It was useful to prevent and cure cervical lesions in Xinjiang Uygur women through high-risk HPV test and DNA ploidy analysis. The transient infection and persistent infection in Xinjiang Uygur women should be taken as further research.

Keywords: Uygur, cervical epithelial cell, human papillomavirus, DNA ploidy analysis

\section{Introduction}

Xinjiang Uygur Autonomous Region is located in the northwest of the People's Republic of China, which is the largest province in the People's Republic of China covering 1.66 million square kilometers with a population of 21.8 million in 2011 . According to the latest census data from the National and Xinjiang Local Statistics Bureau (2011Y), the total population of the People's Republic of China is 1.37 billion, of whom $91.51 \%$ are Han ethnic and $0.76 \%$ are Uygur ethnic, and Xinjiang has a much larger percentage of Uygur ethnic (45\%) and a smaller percentage of Han ethnic (41\%) than in the entire People's Republic of China. The Uygur is one of the indigenous people in the Xinjiang Uygur Autonomous Region of the People's Republic of China. Many diseases in Uygur have particularity because of their custom and genes.

Cervical cancer occurs in the womb malignant tumors of the vagina and cervix tube. More than about 200,000 women die from cervical cancer every year worldwide. 
In the developing countries, cervical cancer has the highest incidence in gynecological tumors. There are 131,500 new cases in the People's Republic of China per year; the general trend is higher incidence in rural than urban area. Most regions in Xinjiang are economically underdeveloped rural areas, so the previous studies reported that the Xinjiang region had the highest incidence of cervical cancer in the entire People's Republic of China. ${ }^{1}$ Especially in the local Uygur women, the morbidity was from 459/100,000 to $590 / 100,000$ and the lethality of cervical cancer was $15.78 / 100,000 .^{2}$ The cervical cancer risk factors, such as women's low social status, poor sexual and menses health, early marriage, married many times, low levels of education, and especially, the female sexual disorder and so on, led to high incidence in the Uygur women. ${ }^{3}$ It is recently reported that there is a trend of a higher incidence of cervical carcinoma in younger women in Xinjiang Uygur. ${ }^{4}$ Therefore, there was important social value to research on the occurrence and prognosis of cervical lesions in Xinjiang Uygur women.

Current studies suggested that cervical cancer was a kind of infectious disease because of human papillomavirus (HPV). It needs 10 years from cervical precancerous lesions to cancer; it is also a relatively longtime continuous development process. HPV16 type was the first infection type in Xinjiang Uygur women, with HPV18 type in the second place, but was significantly lower than other regions. HPV68 and HPV45 types were ranked third; the distribution of subtypes was also different from domestic and international. ${ }^{5}$ All of the above showed that HPV infection in the Xinjiang region had local ethnic characteristics.

DNA ploidy as an objective technology played an important role in the progression of squamous cells cervical cancer. ${ }^{6,7}$ David Garner reviewed the clinical application of DNA ploidy to cervical cancer screening in the People's Republic of China from 2005 to 2013. He reviewed it should has some benefits to combining high-risk HPV testing with DNA ploidy for cervical cancer diagnosis. ${ }^{8}$ In addition, DNA ploidy became an important prognosis factor for cervical lesions or cervical cancer. ${ }^{9,10}$ It should evaluate the therapeutic effects of HPV from two aspects, including host cells and HPV infection. So we speculated that DNA ploidy should also be an important prognosis factor for the cure of HPV infections.

Because HPV infection in Xinjiang region has local ethnic characteristics, there was less research on the DNA ploidy and the HPV infection in Uygur women. So in this paper, we studied the relationship between HPV infection status and DNA ploidy of cervical exfoliated epithelial cells in Xinjiang
Uygur women, in order to contribute to the treatment evaluation of HPV infection in Xinjiang Uygur women.

\section{Materials and methods Sample source}

The sample cases sourced from Xinjiang Uygur women, who initially visited the gynecology department of the tumor hospital affiliated to Xinjiang Medical University from April 2011 to March 2013. We collected the remnant cytology specimens from Liquid-based ThinPrep Cytology Test (TCT); at the same time, we collected the HPV types' test results. A total of 341 cases were collected, but 15 cases were ruled out because the remnants cytology specimens were insufficient or polluted, which did not meet the requirements of the flow cytometry DNA ploidy analysis. According to the inclusion criteria, 326 cases were selected at last. Some patients were also requested to leave contact information to provide convenience for follow-up. The ethics committee of the tumor hospital affiliated to Xinjiang Medical University approved the study and the consent procedure.

\section{Sample collection}

The samples of flow cytometry DNA ploidy analysis from TCT remnants cytology specimens were collected by clinicians according to the specimen collection requirements of TCT. The specimens of HPV typing test were also collected as required by clinicians.

\section{Reagents and instruments}

The HPV typing test used the 21 HPV GenoArray Diagnostic Kit from ChaoZhou Hybribio Biological Chemical Co. Ltd., People's Republic of China. The method of HPV type test is flow-through hybridization and gene chip (FHGC); the equipments for the test such as Thermal Cycler and HybriMax ${ }^{\circledR}$ devices (Flow-through Hybridization HybriMax, US Patent 5,741,647/6,020,187) and so on were also from the ChaoZhou Hybribio Biological Chemical Co. Ltd. The DNA ploidy analysis kit is from the Beckman Coulter; the flow cytometer is Beckman CytomicsTM FC 500. The DNA cell cycle analysis software is also from Beckman Coulter.

\section{Experimental procedure}

Flow cytometry DNA ploidy analysis by TCT remnants cytology specimens:

1. Collected the remnant exfoliative cytology specimens of TCT, which were in cell preservation solution. 
Then through the 300 mesh nylon mesh filter, 1,500 r/s centrifugal for 10 minutes, discarded the liquid supernatant and then repeated this process by adding PBS fluid to the sediment; finally, suspended the exfoliated cells with $1 \mathrm{~mL}$ phosphate buffered saline (PBS) solution.

2. Added $200 \mu \mathrm{L}$ DNA-Prep LPR reagent into the above solution that blended immediately, and placed it for 5 seconds; then added $2 \mathrm{~mL}$ of the DNA Prep Stain reagent into it. Incubated for 20 minutes in dark place. Lastly, tested the specimen by Flow Cytometry DNA Ploidy Analysis System of FC500 flow cytometer.

3. Applied the DNA ploidy analysis software (DNA cell cycle analysis software) to analyze the results and obtained the DNA index (DI) and S-phase cells' peak percentage (SPF) of each specimen.

DNA ploidy analysis is shown in Figure 1.

\section{FHGC for HPV types' testing}

HPV types' testing was carried out by the steps of HPV GenoArray Diagnostic Kit, which could detect 21 HPV genotypes, including $6,11,16,18,31,33,35,39,42,43$, $44,45,51,52,53,56,58,59,66$, and 68 types, and CP8304 types, of which 16, 18, 31, 33, 35, 39, 45, 51, 52, 56, 58, 59, and 68 types are high-risk types.

1. Extracted the HPV viral DNA by DNA extraction kit.

2. Took $1 \mu \mathrm{L}$ of extracted DNA solution and then did PCR amplification according to the instructions in the reaction system by PCR amplification.

3. Made diversion hybridization amplification for amplified DNA samples.

4. Made hybridization results analysis of hybrid membrane after coloration; corresponding color parts' classification is the result.

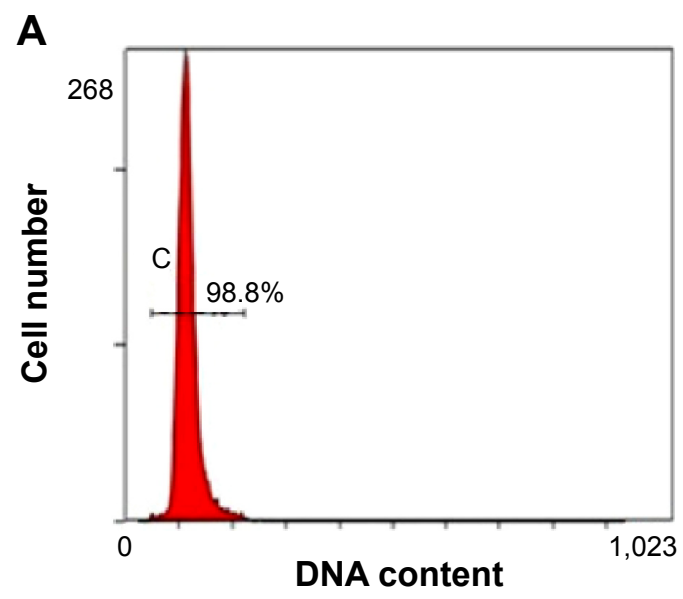

Figure I DNA ploidy of cervical epithelial cells.

Notes: (A) Normal and (B) abnormal DNA ploidy of cervical epithelial cells.

\section{Statistic analysis}

The result was showed by $(\bar{x} \pm s)$ if the data were normally distributed; the statistical analysis was processed by SPSS 18.0 software. For comparison between multiple sets of measurement data using single factor analysis of variance, the Dunnett- $t$ method was used if the measurement has statistical differences. Comparison of count data models was by chi-square test. $\alpha=0.05$ is the inspection level, and $P<0.05$ was received as having statistical differences.

\section{Results}

\section{The HPV infection situation of 326 cases}

A total of 53 specimens were negative in 326 cases. The remaining 273 cases included 21 non-high-risk cases and 252 high-risk-type and multiple infection cases. The detail result is given in Table 1.

\section{The DNA ploidy in different types of HPV infection}

The 326 cases specimens were divided into four groups according to the different types of HPV infection: negative group, non-high-risk infection group, single high-risk-type infection group, and multiple high-risk infection group (contains at least one kind of high-risk infection). Then statistical analysis is carried out in order to find the difference of the DNA ploidy results in different groups. The results are shown in Table 2.

\section{The relationship between HPV infection and DNA ploidy}

$\mathrm{DI}=1.10$ is the threshold of the DNA ploidy results; if a sample's DI was $>1.10$, it was seen as positive in DNA ploidy analysis, which meant heteroploid. If not, the sample

B

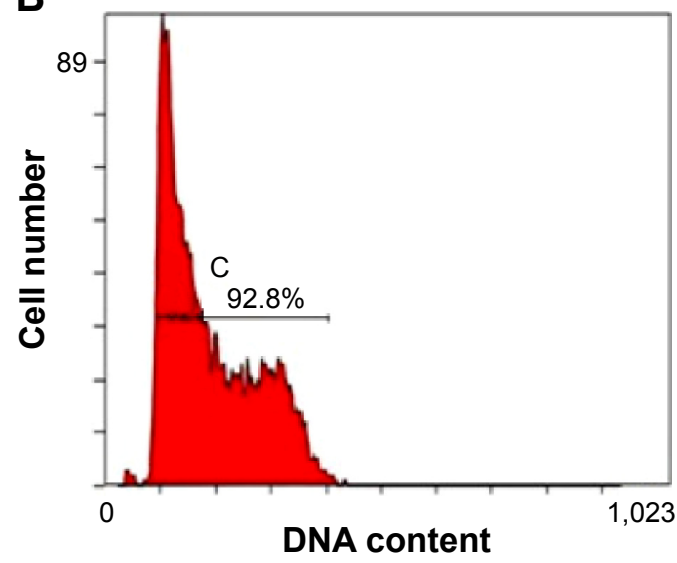


Table I The situation of HPV infection in 326 cases

\begin{tabular}{lll}
\hline & Number & Rate (\%) \\
\hline Negative HPV infection & 53 & 16.3 \\
Non-high-risk HPV infection & 21 & 6.5 \\
53 type & 9 & 2.7 \\
II type & 5 & 1.5 \\
66 type & 4 & 1.2 \\
6 type & 2 & 0.6 \\
44 type & 1 & 0.3 \\
Single high-risk HPV infection & 114 & 35.0 \\
16 type & 81 & 24.8 \\
18 type & 21 & 6.4 \\
45 type & 5 & 1.5 \\
68 type & 3 & 0.9 \\
56 type & 2 & 0.6 \\
59 type & 2 & 0.6 \\
Multiple HPV infection & 138 & 42.3 \\
16/I8 type & 126 & 38.7 \\
16/56 type & 4 & 1.2 \\
16/42 type & 3 & 0.9 \\
16/I8/68 type & 2 & 0.6 \\
16/I8/59 type & 2 & 0.6 \\
16/39 type & 1 & 0.3 \\
\hline A6 & &
\end{tabular}

Abbreviation: HPV, human papillomavirus.

was seen as negative in DNA ploidy analysis. The correlation between the positive rate of DNA ploidy analysis and HPV infection situation is shown in Table 3 .

Compared with the negative group, the DNA heteroploid rate in the non-high-risk-type HPV infection group had no difference $(P=0.135)$. The odds ration (OR) value of heteroploid from single high-risk HPV infection to multiple high-risk HPV infection increased from 12.750 to 22.705 . This means that along with the deepening of HPV infection, the heteroploid rate also increased. The result of trend chi-square test also shows the trend changes.

\section{The DNA ploidy changes of HPV infection patients}

If a patient is infected by HPV, two methods are included. If the patient is infected by non-high-risk HPV, the doctor

Table 2 Comparison of the result of DNA ploidy in different types of HPV infection

\begin{tabular}{llll}
\hline & Number & DI* & SPF* \\
\hline Negative HPV infection & 53 & $\mathrm{I} .00 \mathrm{I} \pm 0.049$ & $4.282 \pm 0.433$ \\
Non-high-risk HPV infection & $2 \mathrm{I}$ & $\mathrm{I} .023 \pm 0.083$ & $4.675 \pm 0.766$ \\
Single high-risk HPV infection & $\mathrm{II} 4$ & $\mathrm{I} .064 \pm 0.088$ & $5.015 \pm 1.049$ \\
Multiple high-risk HPV infection & $\mathrm{I} 38$ & $\mathrm{I} .1 \mathrm{I} \pm \pm 0.085$ & $6.376 \pm 3.368$ \\
$F$ & & 30.775 & 13.940 \\
$P$ & & $<0.00 \mathrm{I}$ & $<0.00 \mathrm{I}$ \\
\hline
\end{tabular}

Notes: *LSD-t: each of negative HPV infection/non-high-risk HPV infection/single high-risk HPV infection and multiple high-risk HPV infection has statistical differences $(P<0.00 \mathrm{I})$. Also statistical differences between single high-risk HPV infection and negative HPV infection exist $(P<0.05)$.

Abbreviations: HPV, human papillomavirus; DI, DNA index; SPF, S-phase cells' peak percentage. usually calls for his/her detection again every 6 months. During follow-up, patients must pay attention to their lifestyle, such as sex life, personal hygiene, and so on. If the patient is infected by single high-risk HPV or multiple high-risk HPV, the doctor usually suggests him/her to take drug therapy and pay attention to lifestyle. At the same time, the doctor usually calls for his/her detection again every 3 months. In this study, 113 cases in 273 cases were followed up in 1 year. The DNA ploidy analysis was done again at the end of the 12th month. In single high-risk HPV infection group, there was one patient who had negative HPV infection and DNA heteroploid after follow-up. In multiple high-risk HPV infection group, the same patients were in eight cases. The follow-up result is shown in Table 4.

\section{Discussion}

In the 273 cases of HPV infection positive specimens, the non-high-risk-type infection was in 21 cases, accounting for $7.69 \%$; single high-risk-type infection was in 114 cases, accounting for $41.76 \%$; and multiple high-risk-type infection was in 138 cases, accounting for $50.55 \%$. In this experiment, the multiple high-risk type HPV infection rate was $50.55 \%$. The rate had no difference compared with the previous study in the People's Republic of China (51.4\%). So there was not much difference between the multiple high-risk HPV infection rate of Uygur women and other population in the People's Republic of China. The previous study also reported that 16/18-type mixed infection was the highest in multiple highrisk HPV infection; in this study, in 126 16/18-type mixed infection cases of 138 multiple high-risk HPV infection cases, which accounted for $91.30 \%$, the rate was consistent with the previous report. ${ }^{11}$ There were 219 cases that were infected with 16-type HPV, accounting for $80.22 \%$ in all infected people. So the 16 type is the highest in all genotypes infection, which is consistent with the previous research's report. ${ }^{5}$

Flow cytometry DNA ploidy analysis can use DI and the percentage of SPF to reflect the situation of the specimens in the cell cycle. Higher DI or SPF values mean more active cell hyperplasia. ${ }^{12}$ There is also positive correlation between high-risk-type HPV virus load and DNA ploidy in cervical exfoliated epithelial cells. ${ }^{13}$ The study showed that, whether it was DI value or SPF value, there was no difference between non-high-risk infection group and negative group/ single high-risk group; there existed obvious statistics difference between single high-risk infection/multiple high-risk infection group and negative group. There were differences between multiple high-risk infection group and the other three groups. So high-risk infection, especially, multiple high-risk HPV infection, leads to cervical cell proliferation activity; on 
Table 3 The relationship between HPV infection groups and DNA heteroploid

\begin{tabular}{|c|c|c|c|c|c|c|c|}
\hline \multirow[t]{2}{*}{ Type of HPV infection } & \multicolumn{5}{|c|}{ DNA ploidy } & \multicolumn{2}{|c|}{ Chi-square test } \\
\hline & Positive & Negative & Positive rate (\%) & OR & $95 \% \mathrm{Cl}$ & $\chi_{\mathrm{MH}}^{2}$ & $\mathbf{P}$ \\
\hline Negative HPV infection & 2 & 51 & 3.77 & - & - & - & - \\
\hline Non-high-risk HPV infection & 3 & 18 & 14.29 & 4.250 & $0.656-27.524$ & - & $0.135^{*}$ \\
\hline Single high-risk HPV infection & 38 & 76 & 33.33 & 12.750 & $2.945-55.204$ & 17.355 & $<0.00 I^{* *}$ \\
\hline Multiple high-risk HPV infection & 65 & 73 & 47.10 & 22.705 & $5.316-96.972$ & 31.566 & $<0.001 * *$ \\
\hline
\end{tabular}

Notes: *Compared with the negative group; $P>0.05$, has no statistical significance. ${ }^{* *}$ Compared with the negative group, $P<0.05$, all have statistical significance. Abbreviations: $\mathrm{HPV}$, human papillomavirus; $\mathrm{OR}$, odds ration; $\mathrm{Cl}$, confidence interval.

the other side, non-high-risk HPV infection has little effect on cervical cell proliferation activity.

The existing report considered that high-risk-type HPV infection may be positively associated with the presence of DNA heteroploid. ${ }^{14-16}$ In the study, the DNA heteroploid rates were $33.33 \%$ and $47.10 \%$, respectively, in single high-risk infection and multiple high-risk infection groups, which were far higher than $3.77 \%$ in the negative group and $14.29 \%$ in the non-high-risk infection group. Negative group as reference to calculate the OR value, the OR was 4.250 in non-high-risk infection group, 95\% CI was 0.656-27.524, had no statistically significant. So the non-high-risk-type HPV infection was not a risk factor for DNA heteroploid. But the OR was 12.750 in single high-risk infection group and the $95 \%$ CI was 2.945-55.204; it may take 12.750 times to appear the DNA heteroploid in single high-risk infection patients compared with the negative HPV infection population. Meanwhile, the OR was 22.705 in multiple high-risk infection group and 95\% CI was 5.316-96.972; it may take 22.705 times to appear the DNA heteroploid in multiple high-risk infection patients compared with the negative HPV infection population. Lee et al reported that single high-risk HPV infection led to a 19.9 times increased risk of cervical cancer, and multiple high-risk HPV infection made the risk of cervical cancer to increase 31.8 times. The experiment's results were consistent with the results of Lee et al's. ${ }^{17}$ Because abnormal DNA ploidy happened along with the seriousness of HPV infection, there was reason to think that DNA ploidy could be a prognostic indicator in therapeutic process of HPV infection.

In this study, the DI and SPF had no differences between single high-risk infection group and non-high-risk infection group; but for DNA heteroploid, the single high-risk HPV infection group had higher rate than non-high-risk infection group. This may produce ambiguity; explaining the ambiguity, we speculated that transient infection and persistent infection were the main reasons. Some transient infection existed in single high-risk infection population. The DI and SPF had large distinction in transient single high-risk infection population and persistent single high-risk infection population; the DI and SPF were always lower in transient single high-risk HPV infection than persistent single high-risk HPV infection, so the whole DI and SPF values were decreased because of transient infection, and this created certain influence on the statistical result. For the morbigenous risk analysis, because it was negative rate and positive rate comparisons, the effect was small; the analysis results were more in accordance with the theory. But the whole situation of HPV transient infection and persistent infection in Xinjiang Uygur women had no epidemiological studies to be reported. Previous epidemiological investigation on HPV infection was mostly cross-sectional research; the longitudinal research in accordance with the timeline was rarely reported. Because the transient infection and persistent infection are important for understanding the cervical epithelial lesions, the later epidemiological studies of HPV infection in Xinjiang Uygur will pay attention to the different infection time types, in order to get the basic situation of persistent infection and transient infection in this population.

While following up the 113 patients with HPV infection for 1 year time, it was found that for single and multiple high-risk HPV infection patients, DI, SPF, and heteroploid of cervical exfoliated cells had significant reduction, which meant that the clinical drug therapy for high-risk HPV infection was most effective. Because the specimen of the study was cervical exfoliated cells, DNA ploidy changed with the renewal of cervical cells. Only the DNA ploidy of new cervical exfoliated cell had been normal; the impact of HPV on the cervical cells had been disappeared. If a patient's cervical lesion was cured, the DNA ploidy analysis result must change to normal. The curative effect of HPV should not be only based on the disappearance of HPV, but also DNA ploidy analysis result was normal, which should be the finally cure criterion of HPV injection.

In a sentence, the study researched the relationship between different HPV infections and cervical exfoliated cells' DNA ploidy. It found that high-risk-type HPV infection, especially multiple high-risk-type HPV infection, could 


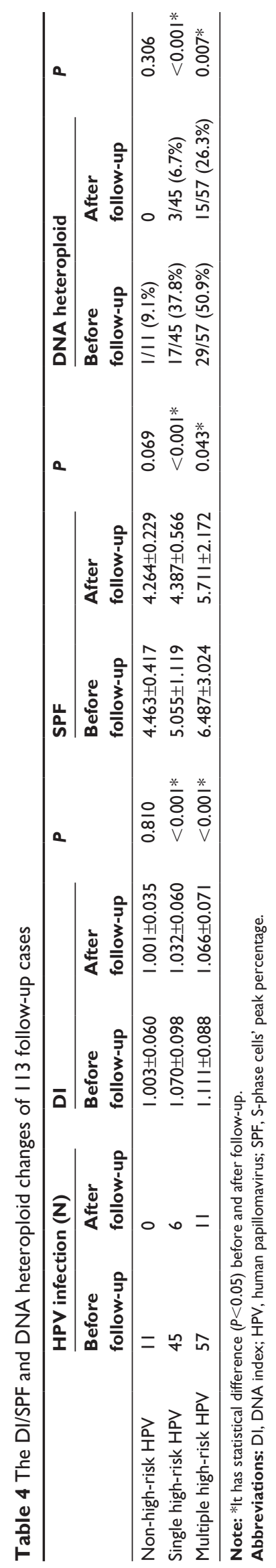

promote cervical cell lesions. Both HPV test and DNA ploidy analysis result were normal, which should be the finally cure criterion of HPV in high-risk-type HPV infection population. Further research should be conducted on transient infection and persistent infection in Xinjiang Uygur women. So it is essential to take high-risk HPV test and DNA ploidy analysis in order to prevent and cure cervical lesions in Xinjiang Uygur women.

\section{Acknowledgment}

We thank Professor Fang Xin-zhi and his colleagues for their assistance in providing the remnants cytology specimens from TCT.

\section{Disclosure}

Potential conflicts do not exist for this study. The authors Yang-Chun Feng, Jia Yang, Zhen-Zhen Cheng, Cheng-Ming Liu, and Yan-Chun Huang declare that they have no conflicts of interest.

\section{References}

1. Chen WB, Huang X, Huang M. Census analysis of 8050 married women about cervical cancer. J Xinjiang Med Univ. 2001;24(3):252.

2. Morgia F, Sbracia M, Schimberni M, et al. A controlled trial of natural cycle versus microdose gonadotropin-releasing hormone analog flare cycles in poor responders undergoing in vitro fertilization. Fertil Steril. 2004; 81(6):1542-1547.

3. Abulafia O, Pezzullo JC, Sherer DM. Performance of thin prep liquidbased cervical cytology in comparison with conventionally prepared Papanicolaou smears: a quantitative survey. Gynecol Oncol. 2003; 90(1):137-144.

4. Zhu ZX, Zhang CL, Zhu JY. The value evaluation of liquid-based cytology test combined with colposcopy examination in diagnosis of cervical intraepithelial neoplasia. Mater Child Health Care China. 2010;25(7): 992-993.

5. Abliz G, Mijit P, Ting-Fang LI. Study on distribution of HPV subtypes in Uygur women with cervical cancer from every region of Xinjiang. J Xinjiang Med Univ. 2009;32(5):513-517.

6. Bollmann R, Méhes G, Torka R, et al. Determination of features indicating progression in atypical squamous cells with undetermined significance: human papillomavirus typing and DNA ploidy analysis from liquid-based cytologic samples. Cancer. 2003;99(2):113-117.

7. Bollmann R, Méhes G, Torka R, et al. Human papillomavirus typing and DNA ploidy determination of squamous intraepithelial lesions in liquid-based cytologic samples. Cancer. 2003;99(1):57-62.

8. David Garner. Clinical application of DNA ploidy to cervical cancer screening: a review. World J Clin Oncol. 2014;5(5):931-965.

9. Pinto AE, Pires A, Silva G, Bicho C, André S, Soares J. Ploidy and S-phase fraction as predictive markers of response to radiotherapy in cervical cancer. Pathol Res Pract. 2011;207(10):623-627.

10. Susini T, Olivieri S, Molino C, et al. DNA ploidy is stronger than lymph node metastasis as prognostic factor in cervical carcinoma: 10-year results of a prospective study. Int J Gynecol Cancer. 2011;21(4): 678-684.

11. Song BZ, Lin MJ, Zhao YM. Clinical analysis of 689 cervical HPV infection cases. Fujian Med J. 2010;32(4):35-37.

12. Singh M, Mehrotra S, Kalra N, Singh U, Shukla Y. Correlation of DNA ploidy with progression of cervical cancer. $J$ Cancer Epidemiol. 2008;2008:1-7. 
13. Feng YC, Zhang Y, Huang YC. The effects of high-risk human papilloma viral load on the DNA ploidy of cervical epithelial cell. J Pathog Biol. 2012;7(1):5-7.

14. Jiao HL, Ye YP, Zhang JL, Li Y, Xia C. Functions of DNA ploidy analysis combined with high-risk human papilloma virus detection in screening of cervical cancer. Mater Child Health Care China. 2010;25(24): 3399-3401.

15. Li XH, Dong WH, Huang ZJ, Zhang ZJ, Wang ZH. The DNA ploidy analysis in precancerous tissue of cervical cancer and human papilloma virus subtype detection. Chin J Obstet Gynecol. 2006;41(3):205-206.
16. Yu XQ, Jiang BL, Fang Y. Combined DNA ploidy analysis and highrisk HPV typing test to predict cervical intraepithelial neoplasia. Chin J Obstet Gynecol. 2013;48(6):459-462.

17. Lee SA, Kang D, Seo SS, et al. Multiple HPV infection cervical cancer screened by HPV DNA chip. Cancer Lett. 2003;198(2):187-192.

\section{Publish your work in this journal}

OncoTargets and Therapy is an international, peer-reviewed, open access journal focusing on the pathological basis of all cancers, potential targets for therapy and treatment protocols employed to improve the management of cancer patients. The journal also focuses on the impact of management programs and new therapeutic agents and protocols on

\section{Dovepress}

patient perspectives such as quality of life, adherence and satisfaction. The manuscript management system is completely online and includes a very quick and fair peer-review system, which is all easy to use. Visit http://www.dovepress.com/testimonials.php to read real quotes from published authors.

\footnotetext{
Submit your manuscript here: http://www.dovepress.com/oncotargets-and-therapy-journal
} 